\title{
Describing Clinical and Histological Outcome of Oral Cancer Patients with Recurrent Malignant or Premalignant Oral Lesions: A Retrospective Series with a Follow-Up of 15 Years ${ }^{+}$
}

\author{
Adriana Cafaro *, Marco Cabras, Alessio Gambino, Marco Garrone, Paolo Giacomo Arduino and \\ Roberto Broccoletti \\ Department of Surgical Sciences, C.I.R. - Dental School, Oral Medicine section, University of Turin, 10126 \\ Turin, Italy; cabrasmarco300@gmail.com (M.C.); alessio.gambino@unito.it (A.G.); ma.garrone@libero.it \\ (M.G.); paologiacomo.arduino@unito.it (P.G.A.); roberto.broccoletti@unito.it (R.B.) \\ * Correspondence: adri.cafaro@gmail.com; Tel.: +39-011-633-15-22 \\ + Presented at the XV National and III International Congress of the Italian Society of Oral Pathology and \\ Medicine (SIPMO), Bari, Italy, 17-19 October 2019.
}

Published: 12 December 2019

\section{Introduction}

Treatment of oral potentially malignant disorders (OPMD) and oral malignant lesions does not simply ends with the complete removal of the affected tissues. Since then, the oral physician plays a key role in terms of secondary prevention, carried out through an appropriate guidance of the patient to a change of lifestyle, and regular post-therapy surveillance. Relapse for head and neck squamous cell carcinoma varies from $16 \%$ to $52 \%$ [2]. Patients with primary tumor diagnosis also have a higher chance to develop second primary tumors (SPTs), arising further than $2 \mathrm{~cm}$ from the primary site and/or 5 years later than primary diagnosis, due to an independent carcinogenesis leading to the onset of a new tumor [1,2]. Aim of the present study was to review retrospectively, from 2003 to 2018, how many patients, firstly diagnosed with an oral malignant neoplasm, experienced a subsequent malignant and/or premalignant disorder, in the form of new OPMD, cancerized OPMD, relapsed cancer or SPT.

\section{Methods}

Electronic archive of the Oral Medicine Section of University of Turin, Italy was reviewed. Inclusion criteria were the following: patients firstly diagnosed with a malignant neoplasm, who later developed at least one OPMD or another malignant neoplasm. The following data were acquired: histological diagnosis, site of occurrence, new OPMD, relapsed OPMD, SPTs and relapsed cancer.

\section{Results}

A sample of 79 patients ( $41 \mathrm{~F}, 38 \mathrm{M}$; mean age 67.5 years) was finally examined, associated with 199 histological reports. Figures 1 and 2 show their distribution based on histology and anatomic site.

Of the 199 lesions detected, $111(55.8 \%)$ were premalignant, whereas 88 (44.2\%) where malignant. Seventy-two of the 88 malignancies $(81.8 \%)$ were diagnosed as oral squamous cell carcinoma (OSCC). Of 199 lesions, 70 could be categorized as relapsed lesions: 38 were diagnosed as OPMD and 32 as neoplasms. Of the remaining 129 new lesions, either detected $2 \mathrm{~cm}$ further from the primary site or 5 years after diagnosis, 62 were SPT, 6 severe OPMD with SPT-like features, and 61 OPMD. 


\section{Discussion}

In this case-series, tongue was by far the most affected site for premalignant and malignant recurrent disorders. Rate of oral cancer relapse in our series aligned with that of the most recent literature ( $36.3 \%$ vs. range of $14-52 \%$ by Netto and co-workers) [2].

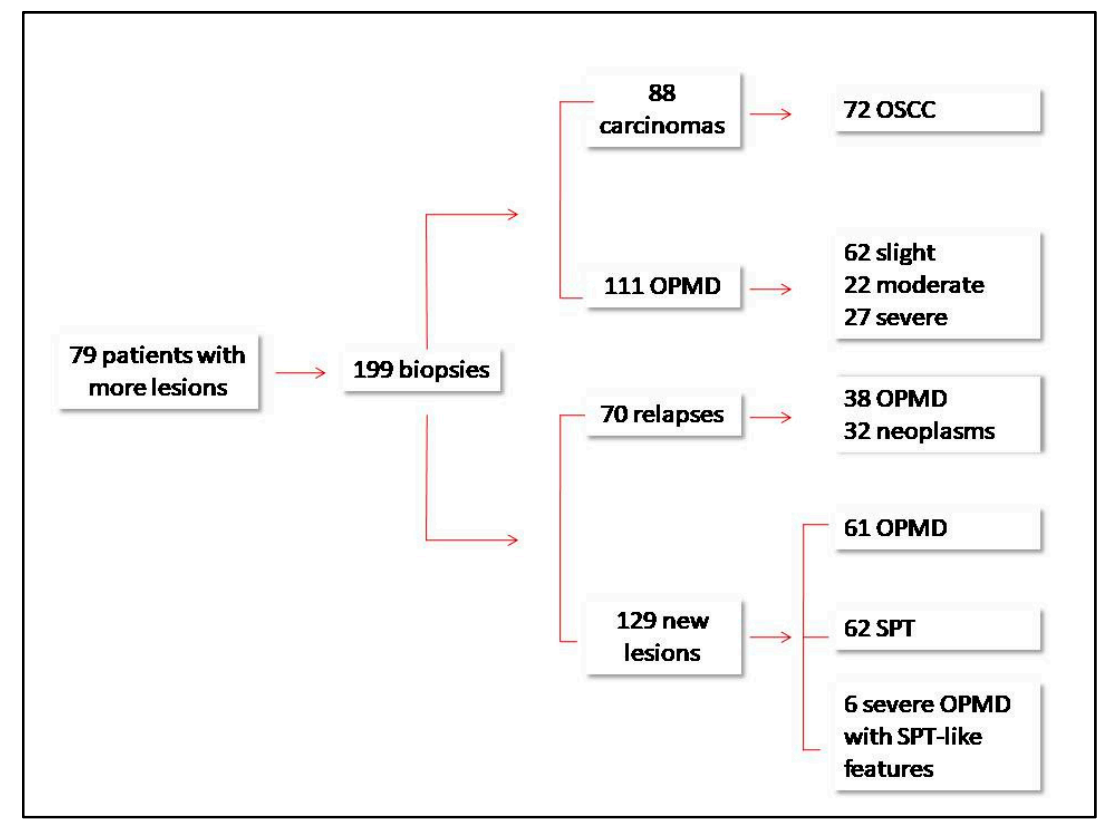

Figure 1. Flow-chart showing the distribution for type of lesion and new lesions/relapses ratio.

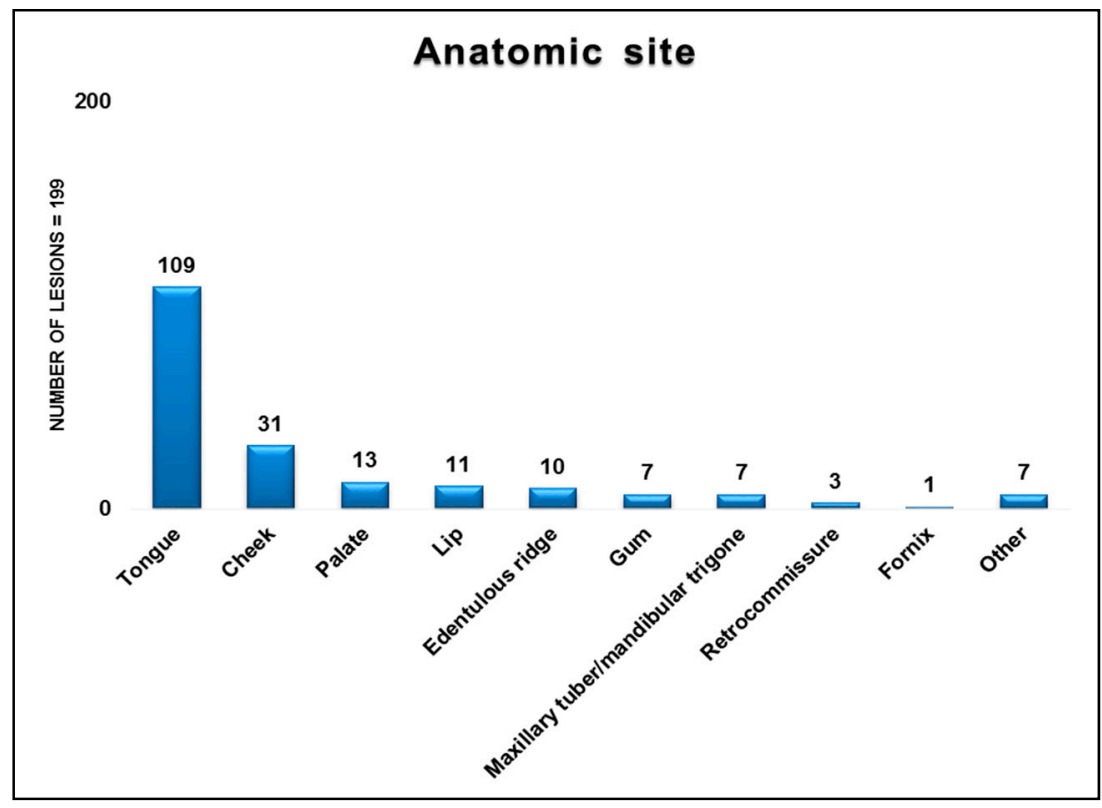

Figure 2. Distribution of the 199 lesions according to the anatomic site. 


\section{References}

1. Brands, M.T.; Smeekens, E.A.J.; Takes, R.P.; Kaanders, J.H.A.M.; Verbeek, A.L.M.; Merkx, M.A.W.; Geurts, S.M.E. Time patterns of recurrence and second primary tumors in a large cohort of patients treated for oral cavity cancer. Cancer Med. 2019, 8, 5810-5819.

2. Gleber-Netto, F.O.; Braakhuis, B.J.; Triantafyllou, A.; Takes, R.P.; Kelner, N.; Rodrigo, J.P.; Strojan, P.; Vander Poorten, V.; Rapidis, A.D.; Rinaldo, A.; et al. Molecular events in relapsed oral squamous cell carcinoma: Recurrence vs. secondary primary tumor. Oral Oncol. 2015, 51, 738-744.

(C) 2019 by the authors. Licensee MDPI, Basel, Switzerland. This article is an open access article distributed under the terms and conditions of the Creative Commons Attribution (CC BY) license (http://creativecommons.org/licenses/by/4.0/). 